SMI-25-95

\title{
The Master Field for QCD and $q$-Deformed Quantum Field Theory
}

\author{
I.Ya.Aref'eva ${ }^{*}$ and I.V.Volovich ${ }^{\dagger}$ \\ Steklov Mathematical Institute, \\ Vavilov 42, GSP-1, 117966, Moscow, Russia
}

\begin{abstract}
The master fields for the large $N$ limit of matrix models and gauge theory are constructed. The master fields satisfy to standard equations of relativistic field theory but fields are quantized according to a new rule. To define the master field we use the Yang-Feldman equation with a free field quantized in the Boltzmannian Fock space. The master field for gauge theory does not take values in a finite-dimensional Lie algebra however there is a non-Abelian gauge symmetry. For the construction of the master field it is essential to work in Minkowski spacetime and to use the Wightman correlation functions. The BRST quantization of the master field for gauge theory and a loop equation are considered.
\end{abstract}

*e-mail: arefeva@arevol.mian.su

$\dagger$ †-mail: volovich@arevol.mian.su 


\section{Introduction}

There exists an old problem in quantum field theory how to construct the master field for the large $N$ limit in QCD. Recently the problem has been reconsidered [1]-[6] by using methods of non-commutative probability theory [7, 8].

The large $N$ limit in QCD where $N$ is the number of colours enables us to understand qualitatively certain striking phenomenological features of strong interactions [9]-12]. To perform an analytical investigation one needs to compute the sum of all planar diagrams. Summation of planar diagrams has been performed only in low dimensional space-time [13, 14].

It was suggested [11] that there exists a master field which dominates the large $N$ limit. The problem of construction of the master field has been discussed in many works, see for example [15]-29]. Recently Gopakumar and Gross [1] and Douglas [2] have constructed the master field for an arbitrary matrix model. The construction requires a knowledge of all correlation functions of a model. In a previous paper [5] we have presented an effective operator realization for the master field (i.e. without knowing an expression for correlation functions) for a subset of planar diagrams, for the so called half-planar diagrams.

The goal of this note is to give an effective operator realization for the master field for all planar diagrams. We construct the master field which satisfies to standard equations of relativistic field theory but it is quantized according to a new rule.

An important fact which we will use is that the master field for a free matrix field is a new free field satisfying to unusual relations $a(k) a^{*}\left(k^{\prime}\right)=\delta\left(k-k^{\prime}\right)$ where $a(k)$ and $a^{*}(k)$ are annihilation and creation operators. Therefore an appropriate functional depending on the free matrix field such as the vacuum expectation of the trace of the product of the Heisenberg field operators goes in the large $N$ limit to a corresponding functional on the new free field. To define the master field we use the Yang-Feldman equation with a free field quantized in the Boltzmannian Fock space.

For a gauge field $A_{\mu}(x)$ we construct the master field $B_{\mu}(x)$. The field $B_{\mu}(x)$ is similar to the quantum photon field in the sense that it does not have matrix indexes. However the commutator $\left[B_{\mu}(x), B_{\nu}(x)\right]$ does not vanish and there is an infinite dimensional non-Abelian operator gauge symmetry for the gauge field $B_{\mu}(x)$ with the gauge group being the group of unitary operators in the Boltzmannian Fock space.

For our construction it is essential to work in Minkowski space-time and to use the Wightman correlation functions [30 and the Yang-Feldman equation in the Heisenberg representation instead of dealing with an Euclidean formulation and using the Feynman correlation functions and the interaction representation.

In Section 2 we will consider the master field for a matrix field model and in Section 3 we will construct the master field for gauge field. We discuss also the BRSTtransformations for general operator fields (not necessary Lie-algebra valued) and con- 
sider the BRST-quantization of the constructed master field for gauge theory and loop equations. We conclude with a discussion of a relation of our master field with a $q$ deformed quantum field theory and with the Adler generalized quantum dynamics.

\section{Scalar field}

Let us consider a model of an Hermitian scalar matrix field $M(x)=\left(M_{i j}(x)\right), i, j=$ $1, \ldots, N$ in the 4-dimensional Minkowski space-time with the field equations

$$
\left(\square+m^{2}\right) M(x)=J(x)
$$

We take the current $J(x)$ equal to

$$
J(x)=-\frac{g}{N} M^{3}(x)
$$

where $g$ is the coupling constant but one can take a more general polynomial over $M(x)$. One integrates eq (2.1) to get the Yang-Feldman equation [31, 32]

$$
M(x)=M^{(i n)}(x)+\int D^{r e t}(x-y) J(y) d y
$$

where $D^{r e t}(x)$ is the retarded Green function for the Klein-Gordon equation,

$$
D^{r e t}(x)=\frac{1}{(2 \pi)^{4}} \int \frac{e^{-i k x}}{m^{2}-k^{2}-i \epsilon k^{0}} d k
$$

and $M^{(i n)}(x)$ is a free Bose field. The $U(N)$-invariant Wightman functions are defined as

$$
W\left(x_{1}, \ldots, x_{k}\right)=\frac{1}{N^{1+\frac{k}{2}}}<0\left|\operatorname{tr}\left(M\left(x_{1}\right) \ldots M\left(x_{k}\right)\right)\right| 0>
$$

where $\mid 0>$ is the Fock vacuum for the free field $M^{(i n)}(x)$.

We will show that the limit of functions (2.4) when $N \rightarrow \infty$ can be expressed in terms of a quantum field $\phi(x)$ (the master field) which is a solution of the equation

$$
\phi(x)=\phi^{(i n)}(x)+\int D^{r e t}(x-y) j(y) d y
$$

where

$$
j(x)=-g \phi^{3}(x)
$$

The master field $\phi(x)$ does not have matrix indexes. Here the free scalar Boltzmannian field $\phi^{(i n)}(x)$ is given by

$$
\phi^{(i n)}(x)=\frac{1}{(2 \pi)^{3 / 2}} \int \frac{d^{3} k}{\sqrt{2 \omega(k)}}\left(a^{*}(k) e^{i k x}+a(k) e^{-i k x}\right)
$$


where $\omega(k)=\sqrt{k^{2}+m^{2}}$. It satisfies to the Klein-Gordon equation

$$
\left(\square+m^{2}\right) \phi^{(i n)}(x)=0
$$

and it is an operator in the Boltzmannian Fock space with relations

$$
a(k) a^{*}\left(k^{\prime}\right)=\delta^{(3)}\left(k-k^{\prime}\right)
$$

and vacuum $\left.\left.\mid \Omega_{0}\right), \quad a(k) \mid \Omega_{0}\right)=0$. A systematical consideration of the Wightman formalism for Boltzmannian fields is presented in [35].

Recall that if $H$ is a Hilbert space then the Boltzmannian Fock space $\Gamma(H)$ over $H$ is just the tensor algebra over $H, \Gamma(H)=\oplus_{n=0}^{\infty} H^{\otimes n}$ (there is no symmetrization or antisymmetrization here). The annihilation and creation operators are defined as

$$
a(f) f_{1} \otimes \ldots \otimes f_{n}=\left(f, f_{1}\right) f_{2} \otimes \ldots \otimes f_{n} ; a^{*}(f) f_{1} \otimes \ldots \otimes f_{n}=f \otimes f_{1} \otimes \ldots \otimes f_{n} .
$$

One has a relation $a(f) a^{*}(h)=(f, h)$ where $(f, h)$ is an inner product in $H$. In our case $H=L^{2}\left(R^{3}\right)$ and one uses notations such as $a(f)=\int a(k) f(k) d k$. An $n$-particle state is created from the vacuum $\left.\mid \Omega_{0}\right)=1$ by the usual formula

$$
\left.\left.\mid k_{1}, \ldots, k_{n}\right)=a^{*}\left(k_{1}\right) \ldots a^{*}\left(k_{n}\right) \mid \Omega_{0}\right)
$$

but it is not symmetric under permutation of $k_{i}$.

Our master field $\phi(x)$ satisfies to the standard equation for the scalar field

$$
\left(\square+m^{2}\right) \phi(x)=-g \phi^{3}(x) .
$$

The only difference of $\phi(x)$ from the standard scalar quantum field is in the relation (2.8). Let us show that the following theorem is true.

Theorem 1. At every order of perturbation theory in the coupling constant one has the following relation

$$
\lim _{N \rightarrow \infty} \frac{1}{N^{1+\frac{k}{2}}}<0\left|\operatorname{tr}\left(M\left(x_{1}\right) \ldots M\left(x_{k}\right)\right)\right| 0>=\left(\Omega_{0}\left|\phi\left(x_{1}\right) \ldots \phi\left(x_{k}\right)\right| \Omega_{0}\right)
$$

where the field $M(x)$ is defined by (2.3) and $\phi(x)$ is defined by (2.5).

To prove the theorem let us expand (2.3) into the perturbative series in the coupling constant $g$,

$$
M(x)=M^{(i n)}(x)+g M^{(1)}(x)+g^{2} M^{(2)}(x)+\ldots
$$

Then at the $n$-th order of the perturbation series one gets a polynomial over $M^{(i n)}$ integrated with a number of propagators $D^{\text {ret }}$,

$$
M^{(n)}(x)=\frac{1}{N^{n}} \int Y^{(n)}\left(x ; y_{1}, \ldots, y_{2 n+1}\right) M^{(i n)}\left(y_{1}\right) \ldots M^{(i n)}\left(y_{2 n+1}\right) d y_{1} \ldots y_{2 n+1}
$$


Here the function $Y^{(n)}$ does not depend on $N$ and it is a polynomial over $D^{r e t}$ and $\delta$-functions. Now let us expand the Wightman functions (2.4) into the perturbative series in the coupling constant $g$,

$$
W\left(x_{1}, \ldots, x_{k}\right)=W^{(0)}\left(x_{1}, \ldots, x_{k}\right)+g W^{(1)}\left(x_{1}, \ldots, x_{k}\right)+\ldots
$$

One has

$$
W^{(n)}\left(x_{1}, \ldots, x_{k}\right)=\frac{1}{N^{1+\frac{k}{2}}} \sum_{|p|=n}<0\left|\operatorname{tr}\left(M^{\left(p_{1}\right)}\left(x_{1}\right) \ldots M^{\left(p_{k}\right)}\left(x_{k}\right)\right)\right| 0>
$$

where $|p|=p_{1}+\ldots+p_{k}$. From (2.11) and (2.12) one gets

$$
\begin{gathered}
W^{(n)}\left(x_{1}, \ldots, x_{k}\right)=\sum_{|p|=n} \int Y^{\left(p_{1}\right)}\left(x_{1} ; y_{1}, \ldots, y_{2 p_{1}+1}\right) \ldots Y^{\left(p_{k}\right)}\left(x_{k} ; z_{1}, \ldots, z_{2 p_{k}+1}\right) \\
\frac{1}{N^{1+\frac{k}{2}+n}}<0\left|\operatorname{tr}\left(M^{(i n)}\left(y_{1}\right) \ldots M^{(i n)}\left(y_{2 p_{1}+1}\right) \ldots M^{(i n)}\left(z_{1}\right) \ldots M^{(i n)}\left(z_{2 p_{k}+1}\right)\right)\right| 0>d y_{1} \ldots d z_{2 p_{k}+1}
\end{gathered}
$$

To perform the limit $N \rightarrow \infty$ we will use the following basic relation

$$
\begin{gathered}
\lim _{N \rightarrow \infty} \frac{1}{N^{1+\frac{k}{2}}}<0\left|\operatorname{tr}\left(\left(M^{(i n)}\left(y_{1}\right)\right)^{p_{1}} \ldots\left(M^{(i n)}\left(y_{r}\right)\right)^{p_{r}}\right)\right| 0> \\
=\left(\Omega_{0}\left|\left(\phi^{(i n)}\left(y_{1}\right)\right)^{p_{1}} \ldots\left(\phi^{(i n)}\left(x_{r}\right)\right)^{p_{r}}\right| \Omega_{0}\right)
\end{gathered}
$$

where $k=p_{1}+\ldots+p_{r}$. To prove (2.14) one uses the Wick theorem for the Wightman functions and 't Hooft's graphs with double lines. One has

$$
<0\left|M_{i j}^{(i n)}(x) M_{p q}^{(i n)}(y)\right| 0>=\delta_{i q} \delta_{j p} D^{-}(x-y)
$$

where

$$
D^{-}(x)=\frac{1}{(2 \pi)^{3}} \int e^{i k x} \theta\left(-k^{0}\right) \delta\left(k^{2}-m^{2}\right) d k .
$$

According to the Wick theorem we represent the vacuum expectation value in the L.H.S. of (2.13) as a sum of 't Hooft's graphs with the propagators (2.15). Then in the limit $N \rightarrow \infty$ only non-crossing (rainbow) graphs are nonvanished. We get the same expression if we compute the R.H.S. of (2.13) by using the relations (2.8), i.e. by using the Boltzmannian Wick theorem. This gives the proof of the relation (2.13).

From (2.13) and (2.14) it follows that

$$
\begin{aligned}
& \lim _{N \rightarrow \infty} W^{(n)}\left(x_{1}, \ldots, x_{k}\right)=\sum_{|p|=n} \int Y^{\left(p_{1}\right)}\left(x_{1} ; y_{1}, \ldots, y_{2 p_{1}+1}\right) \ldots Y^{\left(p_{k}\right)}\left(x_{k} ; z_{1}, \ldots, z_{2 p_{1}+1}\right) \\
& \left(\Omega_{0}\left|\phi^{(i n)}\left(y_{1}\right) \ldots \phi^{(i n)}\left(y_{2 p_{1}+1}\right) \ldots \phi^{(i n)}\left(z_{1}\right) \ldots \phi^{(i n)}\left(z_{2 p_{k}+1}\right)\right| \Omega_{0}\right) d y_{1} \ldots d z_{2 p_{k}+1}
\end{aligned}
$$


Now if one expands equation (2.9) into the perturbative series over the coupling constant $g$,

$$
\phi(x)=\phi^{(i n)}(x)+g \phi^{(1)}(x)+g^{2} \phi^{(2)}(x)+\ldots
$$

then one has

$$
\phi^{(n)}(x)=\int Y^{(n)}\left(x ; y_{1}, \ldots, y_{2 n+1}\right) \ldots \phi^{(i n)}\left(y_{1}\right) \ldots \phi^{(i n)}\left(y_{2 n+1}\right) d y_{1} \ldots d y_{2 n+1}
$$

where functions $Y^{(n)}$ are the same that we have in the expression (2.11) for the expansion of the matrix field $M(x)$ into the perturbative series. Therefore the R.H.S. of (2.16) is equal to

$$
\sum_{|p|=n}\left(\Omega_{0}\left|\phi^{\left(p_{1}\right)}\left(x_{1}\right) \ldots \phi^{\left(p_{k}\right)}\left(x_{k}\right)\right| \Omega_{0}\right),
$$

which is the $n$-th term in the perturbative expansion of the R.H.S. of (2.10) in the coupling constant. This finishes the proof of Theorem 1.

Renormalization of the master field can be performed in the same way as the renormalization of the ordinary Yang-Feldman equation [33, 34, 32]. The renormalized form of the Yang-Feldman equation reads

$$
\Phi(x)=\sqrt{Z} \Phi^{(i n)}(x)+\int D^{r e t}(x-y)\left[g_{R} \Phi^{3}(y)+\delta m^{2} \Phi(y)\right] d y .
$$

The Boltzmannian relations (2.8) can be considered as a special case of a $q$-deformed quantum field theory (see for example [36] - [40], 8, 6] and the references therein) if we quantize a field $\phi(x)$ satisfying to equation (2.9) assuming $q$-commutation relations for the in-field

$$
a(k) a^{*}\left(k^{\prime}\right)-q a^{*}\left(k^{\prime}\right) a(k)=\delta^{(3)}\left(k-k^{\prime}\right)
$$

where $q$ is a parameter. It would be interesting to consider in detail properties of this $q$-deformed model and to see a dependence of renormalization constants on the parameter $q$.

\section{Gauge field}

In this section we construct the master field for gauge field theory. Let us consider the Lagrangian

$$
L=\operatorname{tr}\left\{-\frac{1}{4} F_{\mu \nu}^{2}-\frac{1}{2 \alpha}\left(\partial_{\mu} A_{\mu}\right)^{2}+\bar{c} \partial_{\mu} \nabla_{\mu} c\right\}
$$

where $A_{\mu}$ is the gauge field for the $S U(N)$ group, $c$ and $\bar{c}$ are the Faddeev-Popov ghost fields and $\alpha$ is a gauge fixing parameter. The fields $A_{\mu}, c$ and $\bar{c}$ take values in the adjoint representation. Here

$$
F_{\mu \nu}=\partial_{\mu} A_{\nu}-\partial_{\nu} A_{\mu}+\frac{g}{N^{\frac{1}{2}}}\left[A_{\mu}, A_{\nu}\right], \quad \nabla_{\mu} c=\partial_{\mu} c+\frac{g}{N^{\frac{1}{2}}}\left[A_{\mu}, c\right],
$$


$g$ is the coupling constant. Equations of motion have the form

$$
\begin{gathered}
\nabla_{\mu} F_{\mu \nu}+\frac{1}{\alpha} \partial_{\nu} \partial_{\mu} A_{\mu}+\frac{g}{N^{\frac{1}{2}}} \partial_{\nu} \bar{c} c+\frac{g}{N^{\frac{1}{2}}} c \partial_{\nu} \bar{c}=0 \\
\partial_{\mu}\left(\nabla_{\mu} c\right)=0, \quad \nabla_{\mu}\left(\partial_{\mu} \bar{c}\right)=0
\end{gathered}
$$

One writes these equations in the form

$$
\begin{gathered}
\square A_{\nu}-\left(1-\frac{1}{\alpha}\right) \partial_{\nu} \partial_{\mu} A_{\mu}=J_{\nu}, \\
\square c=J, \quad \square \bar{c}=\bar{J},
\end{gathered}
$$

where

$$
\begin{gathered}
J_{\nu}=-\frac{g}{N^{\frac{1}{2}}} \partial_{\mu}\left[A_{\mu}, A_{\nu}\right]-\frac{g}{N^{\frac{1}{2}}}\left[A_{\mu}, F_{\mu \nu}\right]-\frac{g}{N^{\frac{1}{2}}} \partial_{\nu} \bar{c} c-\frac{g}{N^{\frac{1}{2}}} c \partial_{\nu} \bar{c} \\
J=-\frac{g}{N^{\frac{1}{2}}} \partial_{\mu}\left[A_{\mu}, c\right], \quad \bar{J}=-\frac{g}{N^{\frac{1}{2}}}\left[A_{\mu}, \partial_{\mu} \bar{c}\right]
\end{gathered}
$$

From (3.3) one gets the Yang-Feldman equations

$$
\begin{gathered}
A_{\mu}(x)=A_{\mu}^{(i n)}(x)+\int D_{\mu \nu}^{r e t}(x-y) J_{\nu}(y) d y \\
c(x)=c^{(i n)}(x)+\int D^{r e t}(x-y) J(y) d y, \quad \bar{c}(x)=\bar{c}^{(i n)}(x)+\int D^{r e t}(x-y) \bar{J}(y) d y,
\end{gathered}
$$

where

$$
D_{\mu \nu}^{r e t}(x)=\left(g_{\mu \nu}-(1-\alpha) \frac{\partial_{\mu} \partial_{\nu}}{\square}\right) D^{r e t}(x),
$$

and $g_{\mu \nu}$ is the Minkowski metric. Free in-fields satisfy

$$
\begin{gathered}
\left(\square g_{\mu \nu}-\left(1-\frac{1}{\alpha}\right) \partial_{\mu} \partial_{\nu}\right) A_{\nu}^{(i n)}(x)=0, \\
\square c^{(i n)}(x)=0, \quad \square \bar{c}^{(i n)}(x)=0 .
\end{gathered}
$$

and they are quantized in the Fock space with vacuum $\mid 0>$. The vector field $A_{\mu}^{(i n)}$ is a Bose field and the ghost fields $c^{(i n)}, \bar{c}^{(i n)}$ are Fermi fields. Actually one assumes a gauge $\alpha=1$. In a different gauge one has to introduce additional ghost fields. We introduce the notation $\psi_{i}=\left(A_{\mu}, c, \bar{c}\right)$ for the multiplet of gauge and ghost fields. The $U(N)$-invariant Wightman functions are defined as

$$
W\left(x_{1}, \ldots, x_{k}\right)=\frac{1}{N^{1+\frac{k}{2}}}<0\left|\operatorname{tr}\left(\psi_{i_{1}}\left(x_{1}\right) \ldots \psi_{i_{k}}\left(x_{k}\right)\right)\right| 0>
$$

We will show that the limit of functions (3.5) when $N \rightarrow \infty$ can be expressed in terms of the master fields. The master field for the gauge field $A_{\mu}(x)$ we denote $B_{\mu}(x)$ and 
the master fields for the ghost fields $c(x), \bar{c}(x)$ will be denoted $\eta(x), \bar{\eta}(x)$. The master fields satisfy to equations

$$
\begin{gathered}
D_{\mu} \mathcal{F}_{\mu \nu}+\frac{1}{\alpha} \partial_{\nu} \partial_{\mu} B_{\mu}+g \partial_{\nu} \bar{\eta} \eta+g \eta \partial_{\nu} \bar{\eta}=0, \\
\partial_{\mu}\left(D_{\mu} \eta\right)=0, \quad D_{\mu}\left(\partial_{\mu} \bar{\eta}\right)=0
\end{gathered}
$$

where

$$
\mathcal{F}_{\mu \nu}=\partial_{\mu} B_{\nu}-\partial_{\nu} B_{\mu}+g\left[B_{\mu}, B_{\nu}\right], \quad D_{\mu} \eta=\partial_{\mu} \eta+g\left[B_{\mu}, \eta\right] .
$$

These equations have the form of the Yang-Mills equations (3.3) however the master fields $B_{\mu}, \eta, \bar{\eta}$ do not have matrix indexes and they do not take values in a finite dimensional Lie algebra. The gauge group for the field $B_{\mu}$ is an infinite dimensional group of unitary operators in the Boltzmannian Fock space. Equations (3.6) in terms of currents read

$$
\begin{gathered}
\square B_{\nu}-\left(1-\frac{1}{\alpha}\right) \partial_{\nu} \partial_{\mu} A_{\mu}=j_{\nu}, \\
\square \eta=j, \quad \square \bar{\eta}=\bar{j},
\end{gathered}
$$

where

$$
\begin{gathered}
j_{\nu}=-g \partial_{\mu}\left[B_{\mu}, B_{\nu}\right]-g\left[B_{\mu}, \mathcal{F}_{\mu \nu}\right]-g \partial_{\nu} \bar{\eta} \eta-g \eta \partial_{\nu} \bar{\eta}, \\
j=-g \partial_{\mu}\left[B_{\mu}, \eta\right], \quad \bar{j}=-g\left[B_{\mu}, \partial_{\mu} \bar{\eta}\right] .
\end{gathered}
$$

We define the master fields by using the Yang-Feldman equations

$$
\begin{gathered}
B_{\mu}(x)=B_{\mu}^{(i n)}(x)+\int D_{\mu \nu}^{r e t}(x-y) j_{\nu}(y) d y \\
\eta(x)=\eta^{(i n)}(x)+\int D^{r e t}(x-y) j(y) d y, \quad \bar{\eta}(x)=\bar{\eta}^{(i n)}(x)+\int D^{r e t}(x-y) \bar{j}(y) d y,
\end{gathered}
$$

The in-master fields are quantized in the Boltzmannian Fock space. For the master gauge field we have

$$
B_{\mu}^{(i n)}(x)=\frac{1}{(2 \pi)^{3 / 2}} \int \frac{d^{3} k}{\sqrt{2|k|}} \sum_{\lambda=1}^{4} \epsilon_{\mu}^{(\lambda)}(k)\left[a^{(\lambda) *}(k) e^{i k x}+a^{(\lambda)}(k) e^{-i k x}\right),
$$

where $\epsilon_{\mu}^{(\lambda)}(k)$ are polarization vectors and annihilation and creation operators satisfy

$$
a^{(\lambda)}(k) a^{\left(\lambda^{\prime}\right) *}\left(k^{\prime}\right)=g^{\lambda \lambda^{\prime}} \delta^{(3)}\left(k-k^{\prime}\right),
$$

The expression (3.10) for the field $B_{\mu}(x)$ looks like an expression for the photon field. However because of relations (3.11) the commutator $\left[B_{\mu}(x), B_{\nu}(x)\right]$ does not vanish and it permits us to develope a gauge theory for the field $B_{\mu}(x)$ with a non-Abelian gauge symmetry. 
We quantize the master ghost fields in the Boltzmannian Fock space with indefinite metric

$$
\begin{aligned}
& \eta^{(i n)}(x)=\frac{1}{(2 \pi)^{3 / 2}} \int \frac{d^{3} k}{\sqrt{2|k|}}\left(\gamma^{*}(k) e^{i k x}+\beta(k) e^{-i k x}\right), \\
& \bar{\eta}^{(i n)}(x)=\frac{1}{(2 \pi)^{3 / 2}} \int \frac{d^{3} k}{\sqrt{2|k|}}\left(\beta^{*}(k) e^{i k x}+\gamma(k) e^{-i k x}\right),
\end{aligned}
$$

where creation and annihilation operators satisfy

$$
\begin{gathered}
\gamma(k) \gamma^{*}\left(k^{\prime}\right)=\delta^{(3)}\left(k-k^{\prime}\right), \\
\beta(k) \beta^{*}\left(k^{\prime}\right)=-\delta^{(3)}\left(k-k^{\prime}\right) .
\end{gathered}
$$

We also assume that the product of any annihilation operator with a creation operator of a different type always is equal to zero, i.e.

$$
\begin{aligned}
\gamma(k) \beta^{*}\left(k^{\prime}\right) & =\beta(k) \gamma^{*}\left(k^{\prime}\right)=a^{(\lambda)}(k) \gamma^{*}(k)=0, \\
a^{(\lambda)}(k) \beta^{*}\left(k^{\prime}\right) & =\gamma(k) a^{(\lambda) *}\left(k^{\prime}\right)=\beta(k) a^{(\lambda) *}\left(k^{\prime}\right)=0 .
\end{aligned}
$$

The Boltzmannian Fock vacuum satisfies

$$
\left.\left.\left.\gamma(k) \mid \Omega_{0}\right)=\beta(k) \mid \Omega_{0}\right)=a^{(\lambda)}(k) \mid \Omega_{0}\right)=0 .
$$

Let us denote $\chi_{i}=\left(B_{\mu}, \eta, \bar{\eta}\right)$ the multiplet of the master fields. The following theorem is true.

Theorem 2. At every order of perturbation theory in the coupling constant one has the following relation

$$
\lim _{N \rightarrow \infty} \frac{1}{N^{1+\frac{k}{2}}}<0\left|\operatorname{tr}\left(\psi_{i_{1}}\left(x_{1}\right) \ldots \psi_{i_{k}}\left(x_{k}\right)\right)\right| 0>=\left(\Omega_{0}\left|\chi_{i_{1}}\left(x_{1}\right) \ldots \chi_{i_{k}}\left(x_{k}\right)\right| \Omega_{0}\right)
$$

where the fields $A_{\mu}(x), c(x)$ and $\bar{c}(x)$ are defined by (3.4) and $B_{\mu}(x), \eta(x)$ and $\bar{\eta}(x)$ are defined by (3.9).

The proof of Theorem 2 is analogous to the proof of Theorem 1. We get relations (3.13) for master fields by taking into account the wrong statistics of the ghost fields.

\section{Operator BRST-transformations}

The set of planar diagrams is invariant under gauge transformations. Therefore the theory of the master fields $B_{\mu}, \eta$ and $\bar{\eta}$ also should be gauge invariant. Actually a more general fact is true. Let be given a system of equations

$$
D_{\mu} \mathcal{F}_{\mu \nu}+\frac{1}{\alpha} \partial_{\nu} \partial_{\mu} B_{\mu}+\partial_{\nu} \bar{\eta} \eta+\eta \partial_{\nu} \bar{\eta}=0,
$$




$$
\partial_{\mu}\left(D_{\mu} \eta\right)=0, \quad D_{\mu}\left(\partial_{\mu} \bar{\eta}\right)=0
$$

where

$$
\mathcal{F}_{\mu \nu}=\partial_{\mu} B_{\nu}-\partial_{\nu} B_{\mu}+\left[B_{\mu}, B_{\nu}\right] ; \quad D_{\mu} \eta=\partial_{\mu} \eta+\left[B_{\mu}, \eta\right],
$$

and $B_{\mu}(x), \eta(x)$ and $\bar{\eta}(x)$ are abstract operator valued functions on space-time taking values in an associative algebra not necessary being the master fields. We do not assume any relations like the Hermitian conjugation or anticommutativity for $\eta$ and $\bar{\eta}$. Let us define an operator BRST-transformations

$$
\begin{gathered}
\delta B_{\mu}=D_{\mu} \eta \epsilon, \\
\delta \eta=\eta^{2} \epsilon, \\
\delta \bar{\eta}=-\frac{1}{\alpha} \partial_{\mu} B_{\mu} \epsilon,
\end{gathered}
$$

where $\epsilon$ is a constant infinitesimal parameter such that

$$
\eta \epsilon+\epsilon \eta=0, \quad \bar{\eta} \epsilon+\epsilon \bar{\eta}=0 .
$$

The following proposition is true.

Proposition. If conditions (4.4) are satisfied then equations (4.1) are invariant under the operator BRST-transformations (4.3).

To prove the proposition one performs straightforward computations and uses relations

$$
\begin{gathered}
\delta \mathcal{F}_{\mu \nu}=\left[\mathcal{F}_{\mu \nu}, \eta\right] \epsilon, \quad \delta\left(D_{\mu} \mathcal{F}_{\mu \nu}\right)=\left[D_{\mu} \mathcal{F}_{\mu \nu}, \eta\right] \epsilon \\
D_{\mu} D_{\nu} \mathcal{F}_{\mu \nu}=-\frac{1}{2}\left[\mathcal{F}_{\mu \nu}, \mathcal{F}_{\mu \nu}\right]=0, \quad \delta\left(D_{\mu} \eta\right)=0 .
\end{gathered}
$$

The master field equations (3.6) are a special case of general operator equations (4.1). Therefore the BRST-invariance of the master field equations (3.6) follows from the proposition.

\section{Concluding Remarks}

We have constructed in this paper the master fields describing the large $\mathrm{N}$ limit for matrix scalar, gauge and fermionic fields. Our method based on the Yang-Feldman equation is a general one and can be applied to any matrix model. It is unusual in modern quantum field theory that to solve a problem one has to work in the physical Minkowski space-time instead of the usually adopted Euclidean formulation. Note also

that the formalism of the Wightman correlation functions is more convenient for the 
consideration of the large $\mathrm{N}$ limit then the usually used formalism of Feynman correlation functions and interaction representation. We can introduce a new chronological product $T_{B}$ for the Boltzmannian master fields such that

$$
\begin{gathered}
\left(\Omega_{0}\left|T_{B}\left(\phi^{(i n)}\left(y_{1}\right) \ldots \phi^{(i n)}\left(x_{k}\right)\right)\right| \Omega_{0}\right)= \\
\lim _{N \rightarrow \infty} \frac{1}{N^{1+\frac{k}{2}}}<0 \mid T \operatorname{tr}\left(\left(M^{(i n)}\left(y_{1}\right) \ldots M^{(i n)}\left(y_{r}\right)\right) \mid 0>\right.
\end{gathered}
$$

Here $T$ is the ordinary chronological ordering. An explicit definition of $T_{B}$ is rather involved. It is similar to an explicit realization of the canonical momenta for the free Euclidean master field [5]. A simple definition is found for half-planar diagrams [5, 49]. The formalism of Green functions and functional integrals is convenient in quantum field theory when we deal with Bose or Fermi statistics, i.e. with a simple operator field algebra with commuting or anticommuting generators. However in the large $N$ limit we meet a more complicated operator field algebra and here it is more convenient to work with the Wightman correlators.

Relations (2.8) have been considered by Greenberg 43 as an example of infinite statistics by Doplicher, Haag and Roberts [44].

There are many interesting problems which deserve a further study. A relation of the present approach with the Haan 15] Euclidean approach and with the Greensite and Halpern [29] Euclidean stochastic approach deserves a study. Douglas [2] has discussed the stochastic formulation of [29] in terms of free variables.

It would be interesting to investigate the BRST-formalism for the master gauge field presented in this paper and to see in detail gauge invariance in the line of consideration of the ordinary gauge theory [45]. One can get a more general gauge invariant theory if instead (3.11) one considers $q$-deformed commutation relations for $i n$-fields:

$$
a^{(\lambda)}(k) a^{\left(\lambda^{\prime}\right) *}\left(k^{\prime}\right)-q a^{\left(\lambda^{\prime}\right) *}\left(k^{\prime}\right) a^{(\lambda)}(k)=g^{\lambda \lambda^{\prime}} \delta^{(3)}\left(k-k^{\prime}\right),
$$

Equations of motion for the master field $D_{\mu} \mathcal{F}_{\mu \nu}=0$ are invariant under gauge transformations $B_{\mu} \rightarrow B_{\mu}^{\omega}=\omega^{*} B_{\mu} \omega+\frac{1}{g} \omega^{*} \partial_{\mu} \omega$ where $\omega=\omega(x)$ is an element of the group $U(\mathcal{F})$ of unitary operators in the Boltzmannian Fock space $\mathcal{F}$. Properties of gauge theory with an infinite dimensional gauge group are different from a familiar gauge theory with a finite-dimensional Lie gauge group. In particular, it is known that all homotopic groups of the group $U(H)$ of unitary operators in a Hilbert space $H$ are trivial, $\pi_{k}(U(H))=0$ for $k=0,1, \ldots$ 46. Note that for the group $U(\infty)$ one has $\pi_{2 k-1}(U(\infty))=Z$ and $\pi_{2 k}(U(\infty))=0$ (R.Bott). An approach to gauge theory adapted for a consideration of an infinite dimensional gauge group has been developed in [47. An investigation of topological properties of the infinite-dimensional gauge group for the master field can be useful for the problem of quark confinement in QCD in the large $N$ limit. 
Another interesting question is about an analogue of the Makeenko-Migdal loop equation [21]. In the present approach we have the following relation

$$
\begin{gathered}
W(C)=\lim _{N \rightarrow \infty} \frac{1}{N}<0\left|\operatorname{tr}\left(P \exp \left\{\frac{g}{N^{\frac{1}{2}}} \int_{C} A_{\mu} d x^{\mu}\right\}\right)\right| 0>= \\
\left(\Omega_{0}\left|P \exp \left\{g \int_{C} B_{\mu} d x^{\mu}\right\}\right| \Omega_{0}\right)
\end{gathered}
$$

Here $P$ is the path ordering operator. Since we deal with the Wightman correlation functions we have the following equation

$$
\Delta_{L} W(C)=0
$$

where $\Delta_{L}$ is the generalized Levy Laplacian, see [41, 42]. Here we ignore the ghost fields. This equation is linear in contrast to the Makeenko-Migdal loop equation.

In this note we have considered the equations of motion for the master field. For the construction of an appropriate action we can use the Adler formalism. Adler 48] has proposed a generalization of quantum mechanics in which a dynamics is formulated on a manifold with non-commuting coordinates, which act as operators on an underlying Hilbert space. In this formalism one can make an operator gauge transformation. If we apply this approach to our master gauge field then we can take the Boltzmannian Fock space as the underlying Hilbert space and consider the following Lagrangian

$$
L=\operatorname{Tr}\left\{-\frac{1}{4} \mathcal{F}_{\mu \nu} \mathcal{F}_{\mu \nu}-\frac{1}{2 \alpha} \partial_{\mu} B_{\mu} \partial_{\nu} B_{\nu}+\bar{c} \partial_{\mu} D_{\mu} c\right\}
$$

Here one assumes a $Z_{2}$-grading in the Boltzmannian Fock space and a trace operator $\operatorname{Tr} \mathcal{O}$ for an operator $\mathcal{O}$ is defined as [48] $\operatorname{Tr} \mathcal{O}=R e \Sigma_{n}\left(n\left|(-1)^{F} \mathcal{O}\right| n\right)$ where $R e$ is the real part, $\{\mid n)\}$ is a complete set of states and the Witten index $(-1)^{F}$ counts fermion number modulo two. Then one can make operator variations of the master fields and to get equations (3.6).

We have constructed the master field however still there is a problem of an analytical investigation of correlation functions of the master field. Just to this problem one reduces the problem of quantitative analysis of the planar approximation for QCD.

\section{ACKNOWLEDGMENT}

The authors are grateful to D.Gross and A.Migdal for stimulating discussions of the problem of construction of the master field. This work is supported in part by International Science Foundation grant M1L000. I.A. is supported in part by Russian 
Foundation for Fundamental Research grant 93-011-147. I.V. is supported in part by Russian Foundation for Fundamental Research grant 93-011-140.

\section{References}

[1] R. Gopakumar and D. Gross, Nucl.Phys. B451 (1995) 379.

[2] M. R. Douglas, Stochastic master field, preprint RU-94-81, hep-th/9411025;

M. R. Douglas, "Large $N$ Gauge Theory - Expansions and Transitions," 1994, lectures at the ICTP, hep-th/9409098

[3] M. R. Douglas and M.Li, Phys. Lett. B348 (1995)360.

[4] I. Singer, Talk at the Congress of Mathematical Physics, Paris (1994).

[5] L.Accardi, I.Aref'eva and I.Volovich, The master field for rainbow diagrams and free non-commutative random variables, hep-th/9402092.

[6] L.Accardi, I.Aref'eva, S.Kozirev and I.Volovich, The Master Field for Large N Matrix Models and Quantum Groups Mod.Phys.Lett.(1995), hep-th/9503041

[7] Voiculescu D., Dykema K.J., Nica A. Free random variables, CRM Monograph Series, Vol. 1, American Math. Soc. (1992);

D.V. Voiculescu, Invent. Math., 104 (1991) 201.

[8] L.Accardi, Y.G.Lu, I.Volovich, Non-Commutative (Quantum) Probability, Master Fields and Stochastic Bosonization, preprint CVV-198-94, hep-th/9412246

[9] 't Hooft G., Nucl.Phys. B72 (1974) 461.

[10] G.Veneziano, Nucl.Phys. B160 (1979) 247.

[11] E. Witten, in: "Recent Developments in Gauge Theories", eds. G. 'tHooft et. al. Plenum Press,New York and London (1980)

[12] I.Ya. Aref'eva and A.A.Slavnov, Lectures in the XIV International School of Young Scientists, Dubna,1980;

A.A.Slavnov, Acta Physica Austriaca, Suppl.XXV (1983) 357.

[13] E. Brezin, C. Itzykson, G.Parisi and J.-B. Zuber, Comm.Math.Phys 59 (1978) 35

[14] A.Migdal, JETP,69(1975)810; A.Rusakov, Mod.Phys.Lett.A5(1990)695.

[15] O. Haan, Z. Physik C6 (1980) 345. 
[16] B.Sakita, Phys.Rev. D21(1980)1067;

A.Jevicki and B.Sakita, Nucl.Phys. B165(1980)511.

[17] K.Bardakci, Nucl.Phys. B178(1981)263.

[18] M.B.Halpern and Schwartz, Phys.Rev. D24 (1981) 2146.

[19] P.Cvetanovic, Phys,Lett. 99B(1981)49;

P.Cvetanovic, P.G.Lauwers and P.N.Scharbach, Nucl.Phys. B203 (1982)385.

[20] I.Ya. Aref'eva, Phys. Lett. 104B (1981) 453.

[21] Yu. Makeenko and A. A. Migdal, Nucl. Phys. B188 (1981) 269

[22] A. A. Migdal, Phys. Rep. 102 (1983) 199.

[23] A.Jevicki and H.Levine, Ann.Phys. 13(1981)61.

[24] A.A.Slavnov, Phys. Lett., 112B (1982) 154

[25] T.Eguchi and H.Kawai, Phys. Rev. Lett. 48 (1982) 1063.

[26] G.Parisi, Phys. Lett. 112B (1982) 463

[27] D.J.Gross and Y.Kitazawa, Nucl. Phys. B206 (1982) 440

[28] I.Aref'eva, Phys.Lett. 124B (1982) 221.

[29] J.Greensite and M.B.Halpern, Nucl. Phys. B211 (1983) 343

[30] R.F.Streater and A.S.Wightman, PCT, spin and statistics and all that, W.A.Benjamin, New York-Amsterdam, 1964.

[31] C.N.Yang and D.Feldman, Phys.Rev.,79 (1950)972

[32] J.D.Bjorken and S.D.Drell, Relativistic Quantum Fields, McGraw-Hill, New York, 1965

[33] F.J.Dyson, Phys.Rev. 82(1951)428

[34] G.Kallen, Arkiv f. Phys. 2(1951)371

[35] L.Accardi, I.Aref'eva and I.Volovich, The Master Field in Euclidean and Minkowski Formulation and Free Non-Commutative Random Variables, in preparation.

[36] D.D.Coon, S.Yu and M.M.Backer, Phys. Rev. D5(1972)1429.

[37] V.V.Kurishkin, Ann.Found.L.de Broglie,5(1980)111.

[38] O.W.Greenberg, Phys.Rev.D43(1991)4111. 
[39] I.Aref'eva and I.Volovich, Phys.Lett.B286(1991)179;

I.V.Volovich, Quantum Group Sheaf, hep-th/9404110.

[40] I.Aref'eva, R.Parthasarathy, K.S.Viswanathan and I.Volovich, Mod.Phys.Lett.A9(1994)689

[41] I.Aref'eva and I.Volovich, in Proc. of International Conference on Generalized functions and their applications in mathematical physics, 1981, Moscow.

[42] L.Accardi, P.Gibilisco and I.Volovich, Russian Journ. of Math.Phys, 2(1994) 235.

[43] O.W.Greenberg, Phys.Rev.Lett. 705(1990)705.

[44] S.Doplicher, R.Haag and J.Roberts, Commun. Math.Phys. 23(1971)199;35(1974)49.

[45] N.Nakanishi, Prog.Teor.Phys.Suppl.,51(1972);

T.Kugo and I.Ojima, Prog.Teor.Phys.Suppl.,66(1979)1;

K.Yokoyama, M.Takeda and M.Monda,Prog.Teor.Phys.,64(1980)1412;

K.Izawa, Prog.Teor.Phys., 88(1992)759;

M.Koseki, M.Sato and R.Endo, Prog.Teor.Phys., 90(1993)111

[46] A.S.Schwarz, Dokl.AN USSR, 154(1964)61;

N.H.Kuiper, Topology,3(1965)19;

M.F.Atiyah, K-theory, Cambridge, Mass.,1965.

[47] R.Cianci, M.Francaviglia and I.Volovich, J.Phys.A:Math.Gen.28(1995)723.

[48] S.L.Adler, Generalized Quantum Dynamics, preprint IASSNS-HEP-93/32;

S.L.Adler, Quaternionic quantum mechanics and quantum fields, Oxford University Press, Oxford, 1994

[49] I.Aref'eva and A.Zubarev, Interaction representation for Free Random Variables as an Approximation for the Large $N$ Master Field, preprint SMI-23-95. 